\title{
TRIMMING DOWN THE WILLMAN 1 dSph*
}

\author{
Michael H. Siegel ${ }^{1}$, Matthew D. Shetrone ${ }^{1}$, and Michael Irwin ${ }^{2}$ \\ ${ }^{1}$ University of Texas, McDonald Observatory, 1 University Station, C1402, Austin, TX 78712, USA; siegel@ astro.as.utexas.edu, shetrone@ astro.as.utexas.edu \\ ${ }^{2}$ Institute of Astronomy, Madingley Road, Cambridge, CB3 OHA, UK; mike@ astr.cam.ac.uk \\ Received 2007 December 28; accepted 2008 March 16; published 2008 May 1
}

\begin{abstract}
Willman 1 is a small low-surface-brightness object identified in the Sloan Digital Sky Survey and tentatively classified as a very low luminosity dSph galaxy. Further study has supported this classification while hinting that it may be undergoing disruption by the Milky Way potential. In an effort to better constrain the nature of Willman 1 , we present a comprehensive analysis of the brightest stars in a $0.6 \mathrm{deg}^{2}$ field centered on the overdensity. Highresolution Hobby-Eberly Terlescope (HET) spectra of two previously identified Willman 1 red giant branch (RGB) stars show that one is a metal-rich foreground dwarf while the other is a metal-poor giant. The one RGB star that we confirm as a member of Willman 1 has a low metallicity $([\mathrm{Fe} / \mathrm{H}]=-2.2)$ and a surprisingly low $\alpha$-element abundance $([\alpha / \mathrm{Fe}]=-0.11)$. Washington $+D D O 51$ photometry indicates that $2-5$ of the seven brightest Willman 1 stars identified in previous studies are actually dwarf stars, including some of the more metal-rich stars that have been used to argue both for an abundance spread and a more metal-rich stellar population than galaxies of similar luminosity. The remaining stars are too blue or too faint for photometric classification. The Washington $+D D O 51$ photometry identifies three potential RGB stars in the field but HET spectra show that they are background halo stars. Time series photometry identifies one apparent variable star in the field, but it is unlikely to be associated with Willman 1. Our wide-field survey indicates that over $0.6 \mathrm{deg}^{2}$, Willman 1 does not have a single RR Lyrae star, a single blue horizontal branch (BHB) star, or a single RGB star beyond its tidal radius. While our results confirm that Willman 1 is most likely a low-luminosity metal-poor dSph galaxy, the possibility remains that it is a tidally disrupted metal-poor globular cluster.
\end{abstract}

Key words: galaxies: abundances - galaxies: dwarf - galaxies: halos - galaxies: individual (Willman 1) - galaxies: photometry

\section{INTRODUCTION}

Willman 1 is a small diffuse low-luminosity object identified by Willman et al. (2005, hereafter W05) from an analysis of the Sloan Digital Sky Survey (SDSS). Its size and luminosity were found to be consistent with both low-luminosity globular clusters (e.g., Palomar 1, E3, AM 4, BH 176, Sau A, and AM 1) and low-luminosity dSph galaxies (see Figure 10 of W05). Further investigation revealed an irregular structure, possible mass segregation and a spatial size too large for Willman 1 to be bound without substantial amounts of dark matter (Willman et al. 2007, hereafter W07). Spectroscopic study (Martin et al. 2007; hereafter M07) showed that Willman 1's red giant branch (RGB) stars appear to form a velocity group at $-12.3 \mathrm{~km} \mathrm{~s}^{-1}$ with a moderate velocity dispersion $\left(4.3 \mathrm{~km} \mathrm{~s}^{-1}\right)$. The latter implies a high (470-700) mass-tolight ratio $(\mathrm{M} / \mathrm{L})$ that would potentially be enough to bind Willman 1 at its present galactocentric distance. Additionally, M07 show an apparent spread in abundance with a mean metallicity of $[\mathrm{Fe} / \mathrm{H}]=-1.5$-the latter being consistent with the abundance estimated from main-sequence (MS) photometry (W07; M07). The combination of abundance spread and high dark-matter content would indicate that Willman 1 is a dSph galaxy - the lowest-luminosity dSph known to be orbiting the Galaxy.

However, if Willman 1 is a dSph galaxy, its properties depart significantly from the established patterns of dSph prop-

\footnotetext{
* Based on observations obtained with the Hobby-Eberly Telescope, which is a joint project of the University of Texas at Austin, the Pennsylvania State University, Stanford University, Ludwig-Maximilians-Universität München, and Georg-August-Universität Göttingen.
}

erties (Figure 1). The $\mathrm{M} / \mathrm{L}$ ratio and $[\mathrm{Fe} / \mathrm{H}]$ abundance of dSph galaxies have been shown to follow the total luminosity (see, e.g., Mateo 1998), a trend used to argue that the $\mathrm{dSph}$ galaxies are of similar mass and origin. M07 noted, and we confirm, that Willman 1's apparent $\mathrm{M} / \mathrm{L}$ ratio is an order of magnitude lower than the linear trend established in Milky Way dSph galaxies-although it is similar to other $\mathrm{dSph}$ galaxies of similar luminosity. Willman 1 is also significantly more metal rich than $\mathrm{dSph}$ galaxies of comparable luminosity.

Simon \& Geha (2007, hereafter SG07) used similar departures from the established $M_{V}-[\mathrm{Fe} / \mathrm{H}]$ and $M_{V}-\mathrm{M} / \mathrm{L}$ trends to argue that the Coma and Ursa Major II dSph galaxies could be the tidally disrupted remnants of larger forebears. However, while Ursa Major II appears to overlap the "orphan stream" (Fellhauer et al. 2007), neither Coma nor Willman 1 is known to overlap a halo stream. This is particularly problematic in the case of Willman 1. If Willman 1's relatively high metallicity reflects its initial, rather than current, luminosity, Figure 1 indicates that Willman 1's tidal stream would contain several thousand times more mass than its core.

In this paper, we add several pieces of information to the previous studies of Willman 1 in an effort to clarify its nature. We improve membership analysis and provide a better constraint on the abundance distribution using optimized spectra of two supposed Willman 1 members and Washington+DDO51 photometry (Section 3.1). We also survey Willman 1 for RR Lyrae stars (Section 3.2) and use wide-field photometry to probe the extended distribution of the putative dwarf (Section 3.3). 


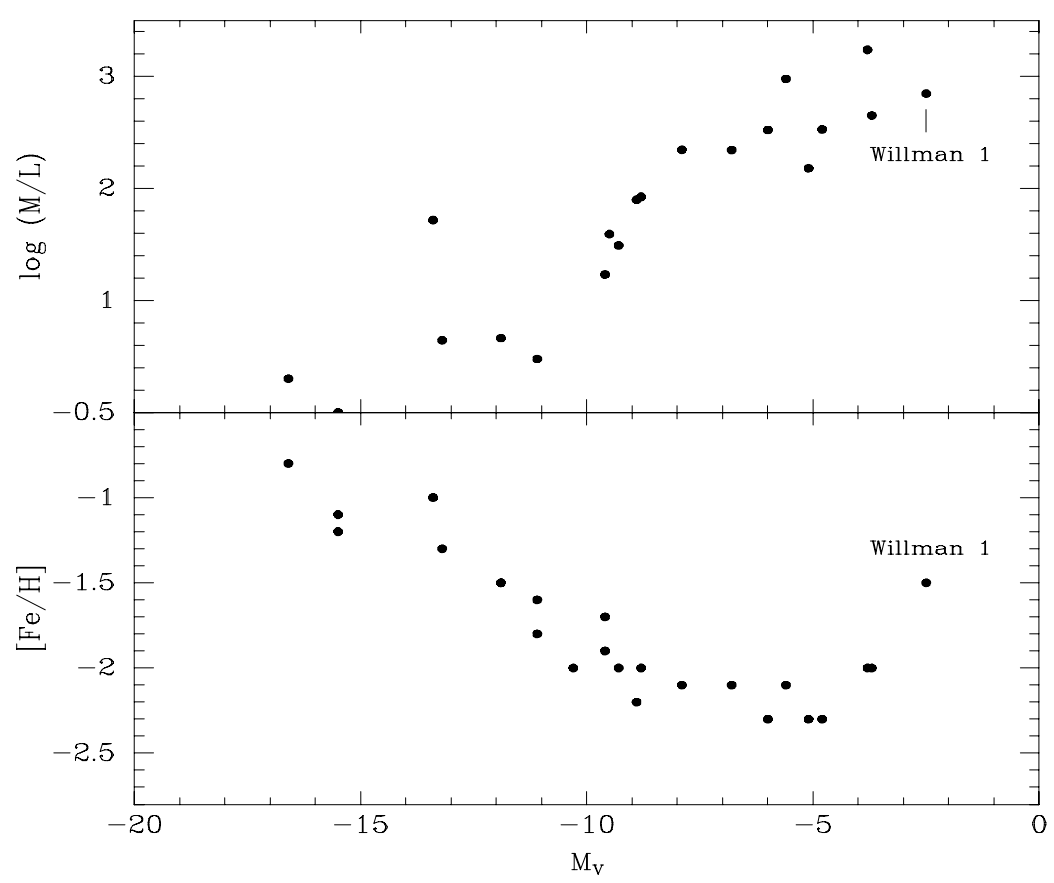

Figure 1. Correlations of the dSph M/L and [Fe/H] to $M_{V}$. Data are taken from Mateo (1998), Muñoz et al. (2006), Zucker et al. (2006), SG07, and M07.

\section{OBSERVATIONS AND DATA REDUCTION}

\subsection{Hobby-Eberly Telescope Spectroscopy}

We obtained spectra of the two brightest Willman 1 RGB stars identified by M07 and three bright RGB candidates identified from Washington photometry (Section 3.1) using the HobbyEberly Telescope (HET) and High-Resolution Spectrograph (HRS, Tull 1998). Data were obtained as part of standard queue scheduled observing (Shetrone et al. 2007) in 2006 November and 2007 April. HRS was configured to achieve $R=18,000$ over the spectral range of 4840-6820 $\mathrm{A}$ with the $600 \mathrm{~g} 5822$ cross disperser. The November data, which were used for abundance analysis, consist of multiple 2250-2700 s exposures taken over several nights to achieve total exposure times of 4500-16200 s and a signal-to-noise ratio $(\mathrm{S} / \mathrm{N})$ of 23-30 per resolution element. The April data, taken to determine radial velocities (RVs), have total exposure times between 600 and $720 \mathrm{~s}$ to achieve $\mathrm{S} / \mathrm{N}>15$ per resolution element.

The spectra were reduced with IRAF's ${ }^{3}$ ECHELLE and TWODSPEC scripts. The standard IRAF scripts for overscan removal, bias subtraction, flat fielding, and scattered light removal were employed. The sky fiber spectra were extracted separately and the two resulting sky spectra were combined and subtracted from the target spectra. We confirmed that no residual solar spectrum remained in the target spectra by cross correlating to the solar atlas of Hinkle et al. (2000) and looking for a peak at zero relative velocity. The target spectra were shifted to the heliocentric rest frame by cross correlating with the Hinkle et al. (2000) Arcturus atlas and combined in the rest frame.

\footnotetext{
3 IRAF (Image Reduction and Analysis Facility) is distributed by the National Optical Astronomy Observatory, which is operated by the Association of Universities for Research in Astronomy, Inc., under cooperative agreement with the National Science Foundation.
}

\subsection{McDonald Observatory $0.8 \mathrm{~m}$ Photometry}

We observed Willman 1 with the McDonald Observatory (MDO) $0.8 \mathrm{~m}$ telescope and Prime Focus Corrector (PFC) on UT 2007 March 19-20 and 2007 April 16-19. The Photometry was obtained in the broadband $B V$, Washington $M T_{2}$ and intermediate-band DDO51 filters. Although unguided, the $0.8 \mathrm{~m}$ telescope is stable enough over $600 \mathrm{~s}$ time intervals to produce excellent wide-field photometry at the coarse (1.3" per pixel) pixel scale of the PFC with little image ellipticity. In an effort to identify any variable stars in the field, we observed ten $B$-band epochs over the course of the observing runs. Total observing times were $6000,600,600,1200$, and $6000 \mathrm{~s}$ in the $B, V, M, T_{2}$, and $D D O 51$ filters, respectively. The 46.0 field of the PFC should easily enclose the entirety of the Willman 1 object, which has a measured half-light radius of $r_{h}=1.9$ and a limiting radius of $\sim 10^{\prime} .0$ (W05, W07, and M07). The PFC covers nearly twice the area of sky as W07's and M07's photometric surveys, although to a much shallower depth.

Data were reduced with the IRAF CCDPROC package and photometered using DAOPHOT (Stetson 1987). The photometry was calibrated to the standards of Landolt (1992) and Geisler $(1990,1996)$ using iterative techniques described in Siegel et al. (2002). Both standard and object stars were measured to total magnitudes using the curve-of-growth analysis of DAOGROW (Stetson 1990). Figure 2 shows the run of photometric error with $V$ magnitude. The line marks the assumed apparent magnitude of Willman 1's horizontal branch (HB) assuming $M_{V}(\mathrm{HB})=$ $0.15[\mathrm{Fe} / \mathrm{H}]+0.80$ and taking the M07 $[\mathrm{Fe} / \mathrm{H}]$ and $(m-M)$ values of -1.5 and 17.9 , respectively. The reddening maps of Schlegel et al. (1998) indicate that the field should have minimal extinction $(E(B-V)=0.01)$. Figure 2 shows that the data provide good $(\sigma \leqslant 0.05)$ photometry in all pass bands for any stars on Willman 1's HB or upper RGB. Our data do not reach the main-sequence turn-off (MSTO) of Willman 1, which would be near $V \sim 22$. 


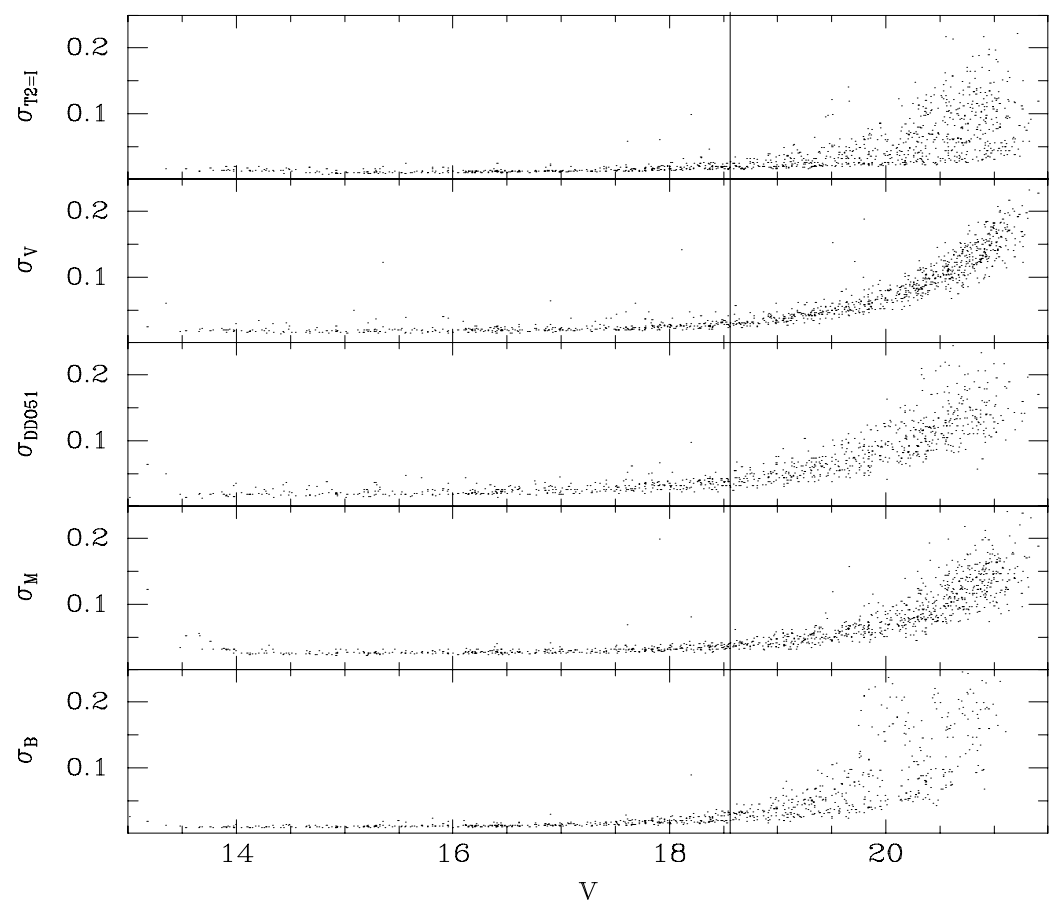

Figure 2. Photometric error in all five pass bands as a function of $V$ magnitude. The line indicates the apparent magnitude of Willman 1's HB, given the properties established in the previous literature.

Accurate stellar positions were measured using the IRAF task TFINDER with the NOMAD astrometric catalog (Zacharias et al. 2004).

\section{THE NATURE OF THE WILLMAN 1 OVERDENSITY}

\subsection{An Improved Membership Survey}

The abundance distribution and velocity dispersion measured by M07 are key arguments for Willman 1's classification as a dSph galaxy and both are inconsistent with the trends established from other dSphs (Figure 1). However, surveys of Willman 1 are hard pressed to separate out bona fide members of such a lowsurface-brightness object from the strong foreground of Milky Way disk stars. RVs are helpful but Willman 1's RV is near the center of the Galactic field star RV distribution (Figure 3), which complicates membership assignment. M07 overcome this difficulty by using the equivalent width of $\mathrm{Na}$ lines as a surface gravity discriminant. However, additional information on stellar surface gravities can be acquired either directly through high$\mathrm{S} / \mathrm{N}$ spectra or indirectly through a photometric proxy.

We used the HET to observe two bright stars classified as Willman 1 members by M07 (1269 and 1578 in our Table $1^{4}$ ) in an effort to determine surface gravities and abundances. The analysis for these two stars used the methods and calibrations of Shetrone et al. (2003) but with the adjustment that the temperature was set to match photometric temperatures derived using the color-temperature relations of Ramirez \& Melendez (2005). Input colors were taken from our own broadband data, the SDSS DR4 (Adelman-McCarthy et al. 2006) and Two Micron All Sky Survey (2MASS) (Skrutskie et al. 2006).

Initial estimates of the surface gravities were made using the distance modulus and reddening of M07. As the two stars differ

\footnotetext{
4 Although the stars we discuss were cataloged by M07, they do not give ID numbers. We refer to these stars by our own ID numbers.
}

in abundance by 2 dex, we confirmed each star's derived surface gravity by requiring the abundance determined from the ionized species to match the abundance determined from the neutral species.

For star 1269, we find an RV of $-11.4 \pm 0.2$, essentially identical to the M07 value of $-10.2 \pm 1.2$. We were able to determine abundances in star 1269 from 25 Fe I lines, 5 Fe II lines, 8 Ti I lines, and 5 Ti II lines. When we fit the spectrum using a $\log g=2.5$ and the temperature derived from near-infrared (NIR) colors $(5350 \mathrm{~K})$, we found large $(3.6 \sigma)$ discrepancies between the Fe I and Fe II abundances for star 1269. The optical colors indicated a lower temperature $(5000 \mathrm{~K})$ but dropping the effective temperature that low only marginally improved the disagreement to $2.4 \sigma$. However, adjusting the surface gravity to that of an MS star $(\log g=4.6)$ brought the $\mathrm{Fe}_{\mathrm{I}}$ and $\mathrm{Fe}$ II abundances to within $1 \sigma$ of each other at a temperature of $5350 \mathrm{~K}$. It is likely that star 1269 is not a Willman 1 object but is a foreground dwarf. We calculate a metallicity of $[\mathrm{Fe} / \mathrm{H}]=-0.18$ under the assumption that this star is a giant - a value significantly more metal rich than the -0.8 measured by M07. We also measure moderate $\mathrm{Mg}$ and $\mathrm{Ca}$ overabundances $([\mathrm{Mg} / \mathrm{Fe}]=+0.21 \pm 0.21,[\mathrm{Ca} / \mathrm{Fe}]$ $=+0.26 \pm 0.11$, respectively), and a significant overabundance of $\mathrm{Ti}([\mathrm{Ti} / \mathrm{Fe}]=+0.76 \pm 0.21)$.

For star 1578, we measure an RV of $-20.47 \pm 1.48$, essentially identical to the $-22.0 \pm 0.6$ measured in M07. We identified $19 \mathrm{Fe}_{\mathrm{I}}$ lines, four Fe II lines, four Ti I lines, and five Ti II lines in the spectrum. Using the photometric temperature (4958 \pm $95 \mathrm{~K}$ in both optical and NIR colors) and an RGB surface gravity $(\log g=2.5)$ produced $1 \sigma$ agreement between the $\mathrm{Fe}_{\mathrm{I}}$ and Fe II abundances, confirming that star 1578 is a low-surfacegravity giant. The measured abundances indicate a metallicity of $[\mathrm{Fe} / \mathrm{H}]=-2.20 \pm 0.17$ with $\alpha$-element abundance ratios slightly below solar $\left(\left[\frac{\mathrm{Mg}+\mathrm{Ca}+\mathrm{Ti}}{3 \mathrm{Fe}}\right]=-0.11 \pm 0.09\right)$. This $\alpha$ abundance would be unusually low for a metal-poor Milky 


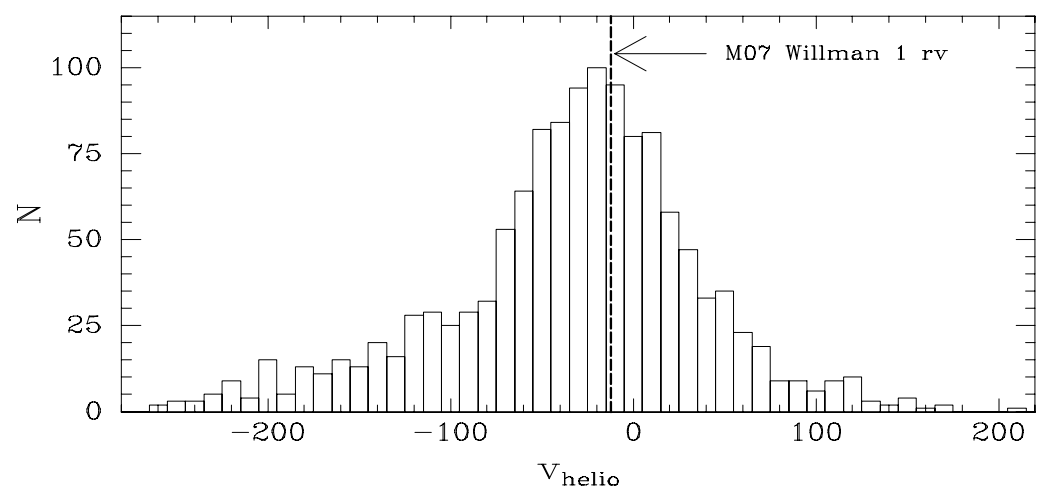

Figure 3. The RV distribution of a theoretical Galactic field star populations in the direction of Willman 1. Galactic simulations were taken from the Besancon model (Robin et al. 2003) and include the thin disk, thick disk, and halo simulated to $V=22$ and out to $50 \mathrm{kpc}$ along the line of sight. The long tail toward negative velocities is the contribution of the thick disk and halo. Willman 1's velocity (solid line) is well within the thin disk peak centered at $-20 \mathrm{~km} \mathrm{~s}{ }^{-1}$.

Way halo star and while the dSph galaxies are known to have low- $\alpha$-abundances, -0.11 would be notably lower than the $\alpha$-abundances of dSph stars of comparable [Fe/H] (see, e.g., Figure 2 of Venn et al. 2004). This would indicate that the much lower luminosity (and low baryonic mass) of Willman 1 has resulted in much slower chemical evolution than the Galactic halo or the dSph galaxies. In that scenario, Willman 1 would be unlikely to the remnant of a larger and therefore a faster-evolving system.

Most of M07's stars are too faint for HET-HRS spectroscopy. However, in the absence of such spectra, the $M-T_{2}-D D O 51$ photometry system can be used to provide a proxy measure of surface gravity that has proven useful in identifying sparse populations of RGB stars against the Galactic foreground (see Majewski et al. 2000a, 2000b; Bessell 2001; Palma et al. 2003;
Westfall et al. 2005; Sohn et al. 2007; Siegel et al. 2008). The three-filter system discriminates high-surface-gravity late-type dwarf stars from low-surface-gravity late-type RGB stars by using the DDO51 filter to measure the strength of the gravitysensitive $\mathrm{MgH}$ and $\mathrm{Mgb}$ absorption features (Paltoglou \& Bell 1994). The $M$ filter provides the necessary continuum measure and, in combination with the $T_{2}=I$ filter, a measure of effective temperature through $M-T_{2}$ color. The referenced studies have shown that this three-filter method is more efficient than simple color-magnitude selection in identifying RGB candidates that are later spectroscopically confirmed. This is especially true at low-surface-brightness levels where the ratio of contaminants is high.

Figure 4 shows the $\left(M-T_{2}, M-D D O 51\right)$ two-color diagrams for the entire survey and the region within 5.0 of the nominal

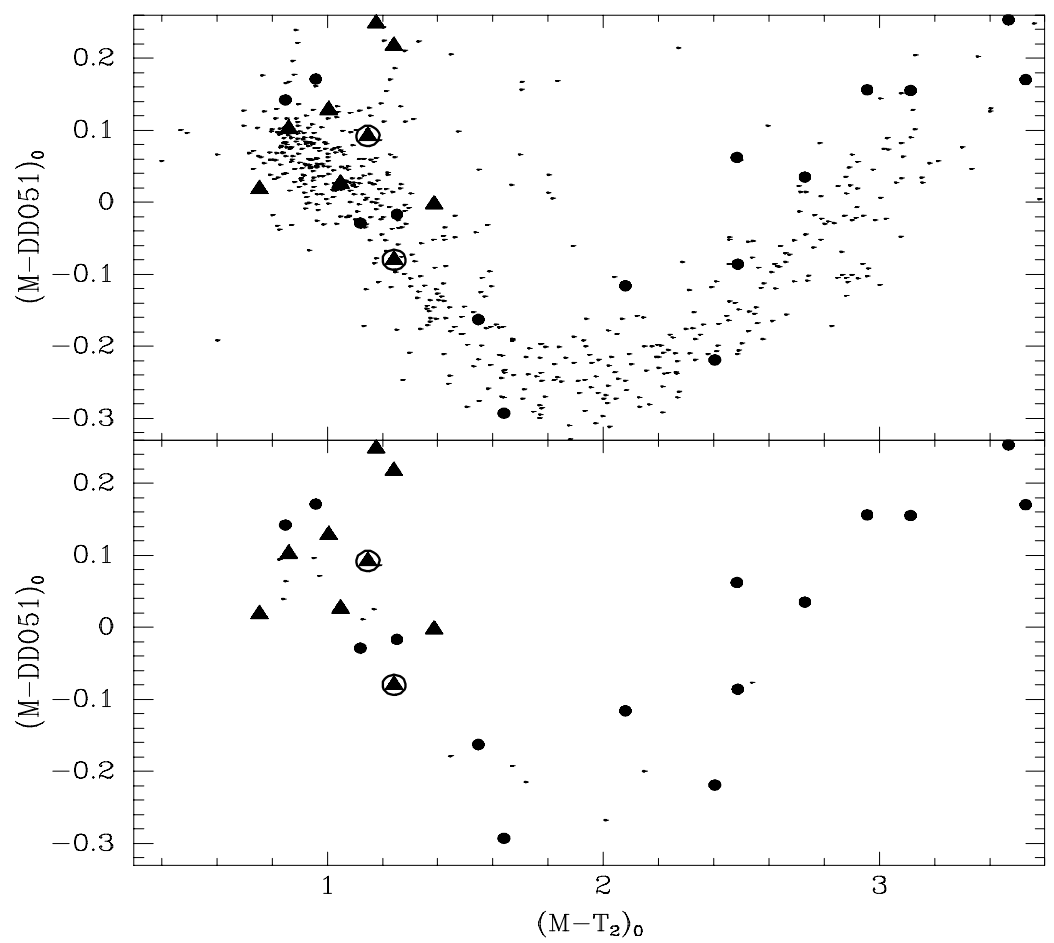

Figure 4. Two-color diagrams of the entire Willman 1 field (top) and the region within $5^{\prime}$ of the nominal photocenter (bottom). The sample is limited to either stars with $-0.1<$ sharp $<0.1$ and $\sigma_{M}, \sigma_{T_{2}}, \sigma_{D D O 51}<0.1$ or to stars matched to the M07 catalog. M07's member stars (triangles) and non-member stars (circles) are marked. The two targets observed with HET-HRS are circled. 
Table 1

Photometric Observations of M07 Spectroscopic Stars

\begin{tabular}{|c|c|c|c|c|c|c|c|c|c|c|}
\hline$\underline{\text { ID }}$ & $(\alpha, \delta)_{2000.0}$ & $M_{0}$ & $\sigma$ & $\left(M-T_{2}\right)_{0}$ & $\sigma$ & $(M-D D O 51)_{0}$ & $\sigma$ & $\Delta_{l}$ & $P_{\text {sRGB }}$ & {$[\mathrm{Fe} / \mathrm{H}]_{\mathrm{M} 07}$} \\
\hline \multicolumn{11}{|c|}{ M07 member stars } \\
\hline 1137 & $10: 48: 58.11,+51: 02: 54.2$ & 21.410 & 0.106 & 1.241 & 0.204 & 0.217 & 0.196 & 0.250 & 0.68 & $\ldots$ \\
\hline 1236 & $10: 49: 08.10,+51: 02: 27.4$ & 21.100 & 0.156 & 1.387 & 0.189 & -0.003 & 0.208 & 0.107 & 0.50 & -1.1 \\
\hline 1269 & $10: 49: 12.41,+51: 05: 44.4$ & 18.791 & 0.038 & 1.242 & 0.042 & -0.080 & 0.056 & -0.021 & 0.04 & -0.8 \\
\hline 1302 & $10: 49: 15.98,+51: 02: 26.9$ & 20.917 & 0.095 & 1.048 & 0.141 & 0.026 & 0.173 & 0.002 & 0.34 & -1.1 \\
\hline 1311 & $10: 49: 17.44,+51: 03: 26.1$ & 20.532 & 0.050 & 1.005 & 0.076 & 0.128 & 0.082 & 0.080 & 0.42 & -1.7 \\
\hline 1334 & $10: 49: 21.18,+51: 03: 30.1$ & 21.027 & 0.137 & 0.860 & 0.213 & 0.102 & 0.177 & 0.014 & $\ldots$ & $\ldots$ \\
\hline 1386 & $10: 49: 27.88,+51: 03: 46.4$ & 20.658 & 0.098 & 0.754 & 0.129 & 0.018 & 0.149 & -0.086 & $\ldots$ & -1.6 \\
\hline 1485 & $10: 49: 40.85,+51: 03: 40.4$ & 21.283 & 0.174 & 1.177 & 0.240 & 0.248 & 0.217 & 0.253 & 0.69 & -1.4 \\
\hline 1578 & $10: 49: 52.55,+51: 03: 42.7$ & 18.602 & 0.038 & 1.147 & 0.045 & 0.092 & 0.054 & 0.098 & 0.46 & -2.1 \\
\hline \multicolumn{11}{|c|}{ M07 non-member stars } \\
\hline 971 & $10: 48: 39.51,+51: 04: 36.0$ & 19.521 & 0.052 & 2.730 & 0.056 & 0.035 & 0.064 & 0.077 & 0.36 & $\ldots$ \\
\hline 1020 & $10: 48: 45.14,+51: 05: 36.4$ & 20.657 & 0.070 & 2.484 & 0.078 & 0.062 & 0.123 & 0.190 & 0.70 & \\
\hline 1028 & $10: 48: 46.15,+51: 02: 12.5$ & 18.975 & 0.035 & 2.080 & 0.040 & -0.116 & 0.051 & 0.128 & 0.64 & $\ldots$ \\
\hline 1074 & $10: 48: 51.59,+51: 03: 10.1$ & 19.744 & 0.056 & 0.848 & 0.072 & 0.142 & 0.073 & 0.051 & $\ldots$ & $\ldots$ \\
\hline 1091 & $10: 48: 53.26,+51: 06: 30.8$ & 21.129 & 0.108 & 2.956 & 0.114 & 0.156 & 0.155 & 0.128 & 0.55 & $\ldots$ \\
\hline 1092 & $10: 48: 53.66,+51: 06: 24.1$ & 21.510 & 0.098 & 3.468 & 0.102 & 0.253 & 0.161 & 0.286 & $\ldots$ & $\ldots$ \\
\hline 1198 & $10: 49: 03.89,+51: 06: 40.4$ & 17.366 & 0.026 & 2.487 & 0.029 & -0.086 & 0.040 & 0.051 & 0.15 & $\ldots$ \\
\hline 1222 & $10: 49: 06.85,+51: 04: 23.7$ & 18.803 & 0.046 & 0.958 & 0.052 & 0.171 & 0.056 & 0.106 & $\ldots$ & $\ldots$ \\
\hline 1437 & $10: 49: 33.86,+51: 03: 33.8$ & 20.631 & 0.061 & 1.640 & 0.075 & -0.293 & 0.118 & -0.082 & 0.12 & $\ldots$ \\
\hline 1496 & $10: 49: 42.90,+51: 04: 23.0$ & 18.726 & 0.037 & 1.252 & 0.042 & -0.017 & 0.045 & 0.041 & 0.12 & $\ldots$ \\
\hline 1616 & $10: 49: 56.99,+51: 05: 49.6$ & 19.055 & 0.040 & 3.113 & 0.043 & 0.155 & 0.055 & 0.109 & $\ldots$ & $\ldots$ \\
\hline 1733 & $10: 50: 01.32,+51: 04: 43.5$ & 21.168 & 0.086 & 3.530 & 0.090 & 0.170 & 0.167 & 0.245 & $\ldots$ & $\ldots$ \\
\hline 1785 & $10: 50: 07.88,+51: 02: 57.6$ & 18.869 & 0.038 & 1.547 & 0.043 & -0.163 & 0.051 & 0.016 & 0.09 & $\ldots$ \\
\hline 1803 & $10: 50: 09.53,+51: 04: 15.2$ & 19.510 & 0.032 & 2.404 & 0.037 & -0.219 & 0.059 & -0.046 & 0.03 & $\ldots$ \\
\hline 1853 & $10: 50: 15.75,+51: 02: 22.5$ & 15.770 & 0.027 & 1.120 & 0.029 & -0.029 & 0.034 & -0.024 & 0.00 & $\ldots$ \\
\hline
\end{tabular}

Willman 1 photocenter. Stars have been selected to be PSF-like $(-0.1<$ sharps $<0.1$; Siegel et al. 2002) or to have small errors $\left(\sigma_{M}, \sigma_{T_{2}}, \sigma_{D D O 51}<0.1\right)$. The long arc of stars at negative $M-D D O 51$ colors are the field dwarfs and any very metal-rich giants. Intermediate to metal-poor RGB stars normally reside in the space inside this curve, clustered near $(M-D D O 51) \sim 0$. However, in contrast to the studies referenced above, there is no prominent RGB in the Willman 1 field. Rather, there are a few stars near $M-D D O 51 \sim 0$ that represent a combination of Willman 1 RGB stars, field RGB stars, background galaxies, and/or foreground metal-poor dwarf stars.

We have marked in Figure 4 the stars that have counterparts in the M07 survey. Our photometric survey matches nine stars that M07 classify as members and 15 stars that they classify as non-members. A preliminary examination of Figure 4 indicates that only two of M07's member stars are clearly RGB stars while most of the non-members and a significant number of member stars have photometry consistent with dwarfs.

However, this initial impression is deceptive. Most of M07's stars are quite faint and the photometric uncertainties for these stars, especially in the critical DDO51 filter, are substantial (median $0.14 \mathrm{mag}$ ). We determined the nature of these stars by first calculating the $\Delta_{l}$ measure as described in Siegel et al. (2008). $\Delta_{l}$ measures the distance of each star orthogonal to a polynomial fit of the dominant dwarf sequence (Figure 5) over the color range $1.0<\left(M-T_{2}\right)_{0}<3.0$. With the problem reduced to a single dimension, we calculate how much of each star's Gaussian probability distribution function lies within the RGB box. ${ }^{5}$

\footnotetext{
5 No color-magnitude selection was applied owing to the sparseness of the Willman 1 RGB.
}

Table 1 lists magnitude, errors, $\Delta_{l}$ measures, and the likelihood that each M07 star in our program has true photometric properties within the RGB locus. Many of M07's stars are close to $50 \%$, indicating that our photometry of these stars is too poor for reliable classification. Additionally, a number of them are too blue, being close to the MSTO where the Mg absorption features vanish. However, we find that seven of M07's member stars are within the $\left(M-T_{2}\right)_{0}$ color range at which $\mathrm{Mg}$ features manifest. Two of these stars have less than a $35 \%$ chance of residing within the RGB color-color locus, including star 1269 , confirming the spectral analysis. Three are ambiguous, with probabilities between $42 \%$ and 50\%, including star 1578 . Two are most likely RGB stars with probabilities greater than $68 \%$.

Of M07's non-member stars, ten are in the usable color range. Six of these stars are clearly dwarfs $(\leqslant 15 \%)$, two are ambiguous ( $36 \%$ and $55 \%$ ), and two are clearly within the RGB locus (>64\%) -although well removed from Willman 1's RGB (Figure 6).

We used our classification methods to identify any RGB stars missed in the M07 study and found three bright RGB candidates $\left(\Delta_{l}>.105 ; P_{\mathrm{RGB}}>0.7 ; M<19\right)$. All three are well outside Willman 1's $r_{h}$ but lie near the hypothetical Willman 1 RGB. The properties of these stars, including the RVs measured with HET-HRS, are listed in Table 2. RV uncertainties are $\sim 1 \mathrm{~km} \mathrm{~s}^{-1}$. As can be seen, none of these three stars lies at the nominal Willman $1 \mathrm{RV}$. They resemble a typical high-dispersion halo population, meaning they are either foreground subdwarfs or unrelated halo RGB stars.

Figure 6 shows the $V I$ color-magnitude diagram (CMD) of the Willman 1 field. The bright stars are taken from the photometric survey described in Section 2.2 while the faint 


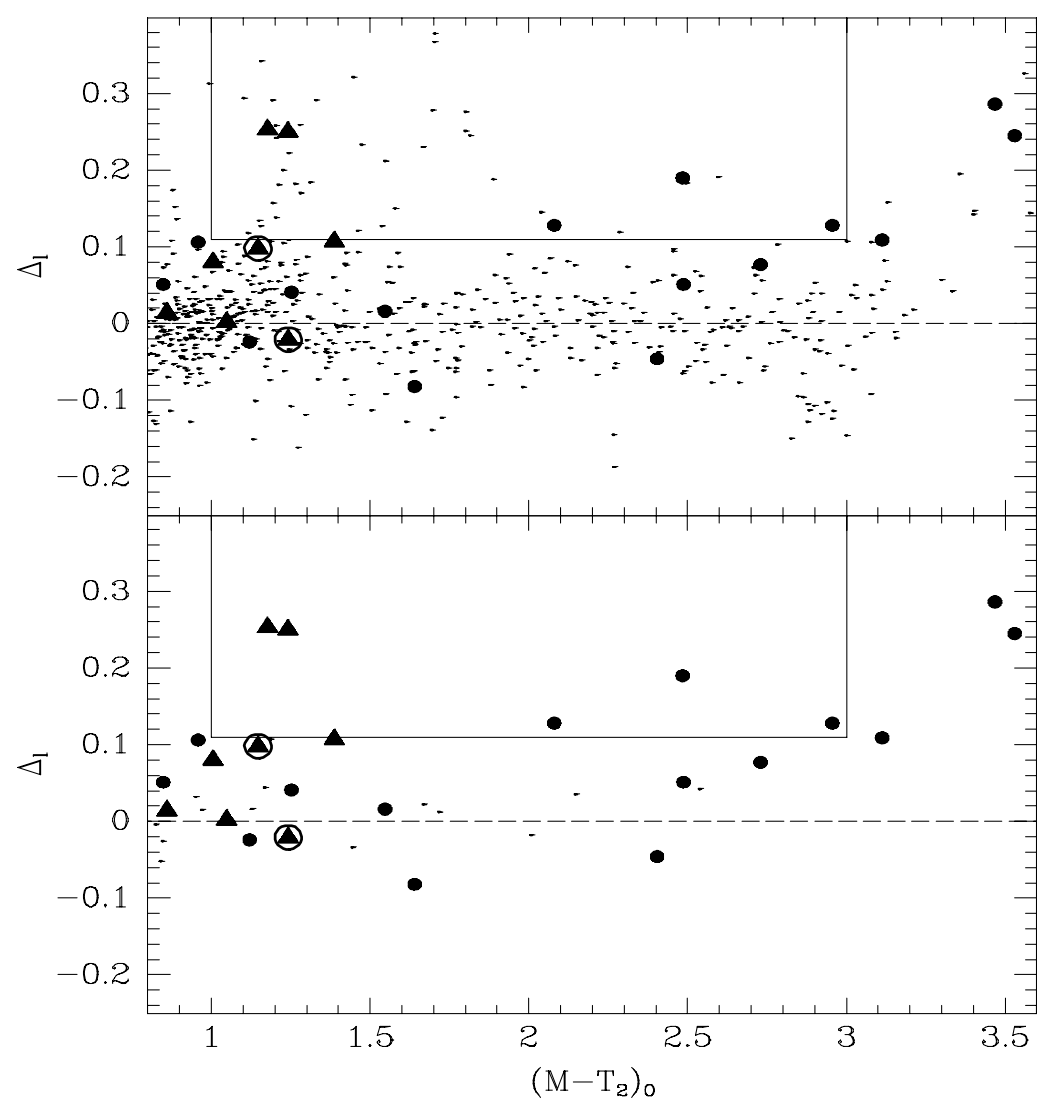

Figure 5. $\Delta_{l}$ diagrams that measure the photometric distance of each star from a fitted dwarf locus over the color range $\left.1.0<\left(M-T_{2}\right)_{0}\right)<3.0$. The sample is limited to either stars with $-0.1<$ sharp $<0.1$ and $\sigma_{M}, \sigma_{T_{2}}, \sigma_{D D O 51}<0.1$ or to stars matched to the M07 catalog. M07's member stars (triangles) and non-member stars (circles) are marked. The two targets observed with HET-HRS are circled. The box marks our RGB selection criteria.

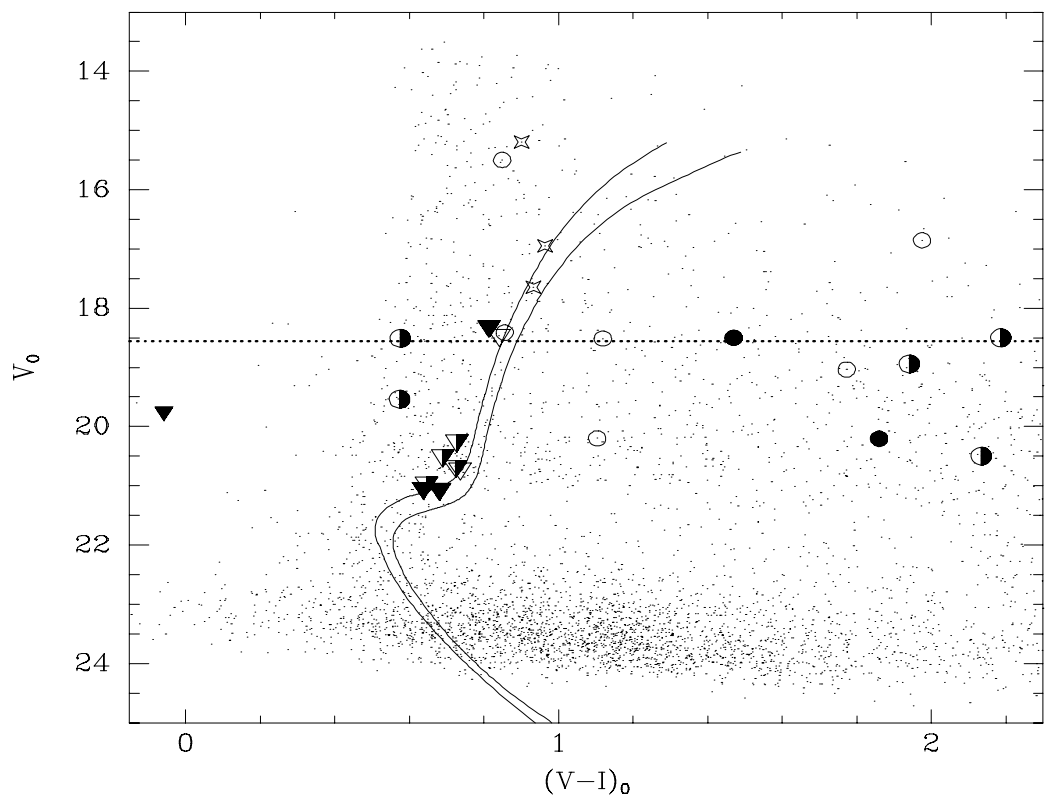

Figure 6. VI CMD of stars surveyed by M07 and this study. Triangles are stars identified as Willman 1 members in M07; circles are those identified as non-members. Empty symbols are those we identify as non-giants; half-filled symbols are ambiguous; filled symbols are those we identify as giants. Star symbols are Washingtonselected RGB candidates observed with HET, all three of which appear to be non-members. The solid lines are theoretical isochrones from Dotter et al. (2007) while the dotted line represents the likely magnitude of the HB. The solid triangle near the HB is the member star identified in W05.

stars are taken from M07's INT-WFC study. The likely HB magnitude is indicated by the dotted line while theoretical isochrones from Dotter et al. (2007) set at $[\mathrm{Fe} / \mathrm{H}]=-1.5$ and $-2.2,[\alpha / \mathrm{Fe}]=+0.0,14 \mathrm{Gyr}$ are overlaid for comparison. Large points indicate RGB likelihood. M07 member stars are marked as triangles; non-members as circles. Filled symbols are those 

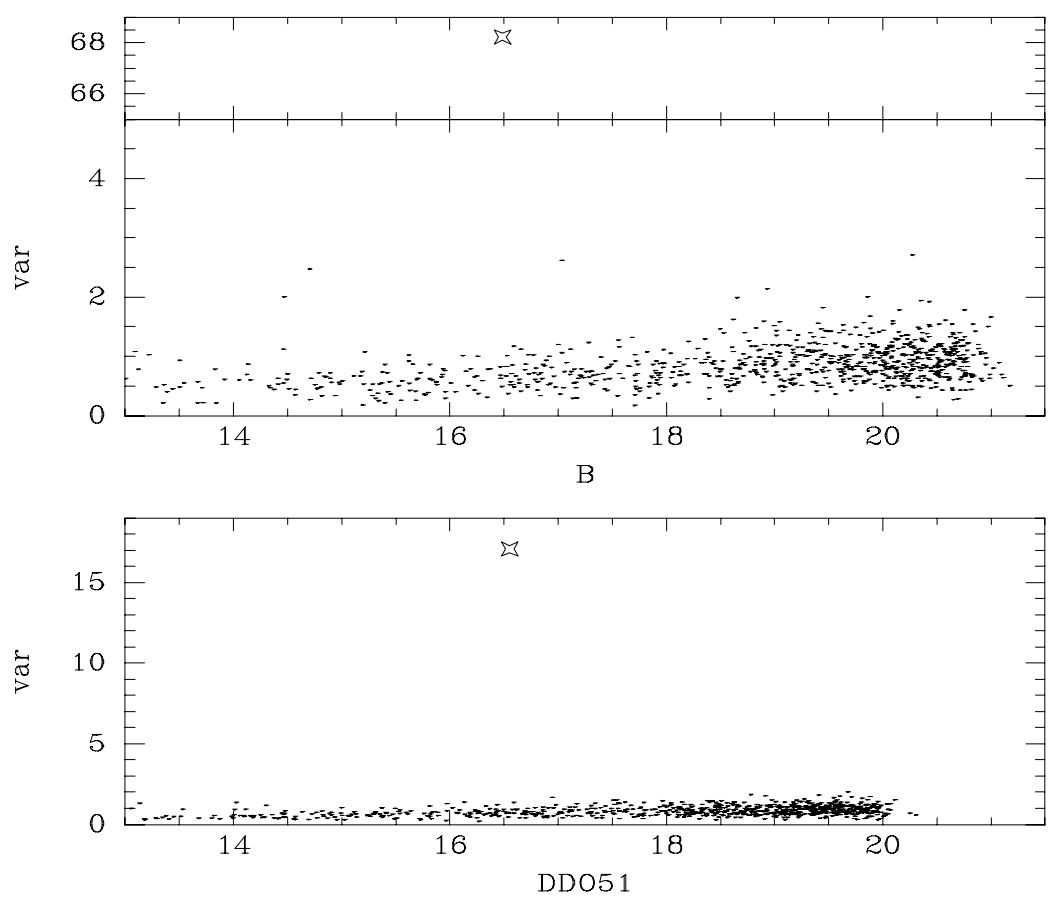

Figure 7. Variability of stars within the Willman 1 field. The starred point indicates the variable star. No other object has a variability greater than three times its formal error.

we identify as RGB stars; half-filled are those either outside the useable range of $\left(M-T_{2}\right)_{0}$ color or with uncertain classification; empty symbols are those we identify as non-RGB stars. The starred points mark the objects identified as potential giants in Table 2 that have RVs inconsistent with Willman 1 membership. We have also marked with a filled triangle the HB star noted by W05 (SDSSJ104913.15+5210232.6). SDSS DR6 indicates this star has an RV of $\sim-20 \mathrm{~km} \mathrm{~s}^{-1}$, making it a likely Willman 1 member (see Section 3.3).

As can be seen, a significant number of M07's stars, including one of their brightest members, disappear from the CMD when the $M-T_{2}-D D O 51$ photometric and HET-HRS information are included. No new members are added by the $0.6 \mathrm{deg}^{2}$ photometric survey. The confirmed member stars lie almost exclusively on the metal-poor isochrone-although a younger metal-rich isochrone would also fit the data.

\subsection{A Search for RR Lyrae Variable Stars}

RR Lyrae variable stars can provide both a precise distance measure to and age/abundance information about their parent stellar population (see, e.g., Siegel 2006). Additionally, RR Lyrae stars can be used as tracers to explore the extended structure of dSph galaxies (see, e.g., Kuhn et al. 1996).

In an attempt to identify variable stars in the Willman 1 field, we obtained ten $B$-band epochs over the course of the two MDO $0.8 \mathrm{~m}$ observing runs. A simple plot of the ratio of measured dispersion over formal photometric error (Figure 7) shows that there is one highly variable star (at a significance of $68 \sigma$ ) in the Willman 1 field with an approximate amplitude of $A_{B} \sim 1.4$. Most other stars vary within $1-2 \sigma$ of their mean values. Of the three remaining stars with a variability greater than twice their formal error, two are bright $(B \sim 14.5)$ and are either saturated or have underestimated formal errors. The remaining star has a single discrepant measure. We ran a similar analysis of the DDO51 data, which consists of ten epochs summed to produced the deep DDO51 image for our photometric analysis (Section 3.1). While these data were obtained in a shorter span of time (two nights) and are therefore less optimized for variablestar searching, they show a similar pattern (lower panel of Figure 7) to the $B$ data and identify the same variable star.

We attempted to fit the light curve using the fitting code and templates of Layden (1998) and Siegel (2006) but found the fit periods to be degenerate. While the data are not extensive enough to break the period degeneracy, they indicate that this variable is unlikely to be associated with Willman 1 . First, the variable star has a mean magnitude of $\langle B\rangle=17.02,2 \mathrm{mag}$ brighter than the likely HB of Willman 1 (see Figure 8). Second, the variable is over $20^{\prime}$ from the nominal Willman 1 center-ten times the $r_{h}$ measured by W05. Although located within $30^{\circ}$ of the apparent Willman 1 major axis (see Figure 3 of W07), it is unlikely that the only variable star in Willman 1 would be so far removed from the photocenter.

Table 2

HET RVs of Washington-Selected Stars

\begin{tabular}{lcccccccccc}
\hline \hline ID & $(\alpha, \delta)_{2000.0}$ & $V_{0}$ & $(B-V)_{0}$ & $(V-I)_{0}$ & $M_{0}$ & $\left(M-T_{2}\right)_{0}$ & $(M-D D O 51)_{0}$ & $\Delta_{l}$ & $P_{\mathrm{RGB}}$ & $v_{\text {hel }}$ \\
\hline 385 & $10: 47: 26.53,+51: 05: 06.8$ & 15.194 & 0.726 & 0.901 & 15.494 & 1.202 & 0.112 & 0.138 & 0.79 & -74.5 \\
849 & $10: 48: 23.16,+51: 19: 20.5$ & 16.954 & 0.819 & 0.964 & 17.269 & 1.280 & 0.112 & 0.170 & 0.93 & -124.0 \\
1304 & $10: 49: 16.56,+51: 14: 20.9$ & 17.651 & 0.779 & 0.933 & 17.981 & 1.263 & 0.133 & 0.182 & 0.94 & +53.7 \\
\hline
\end{tabular}




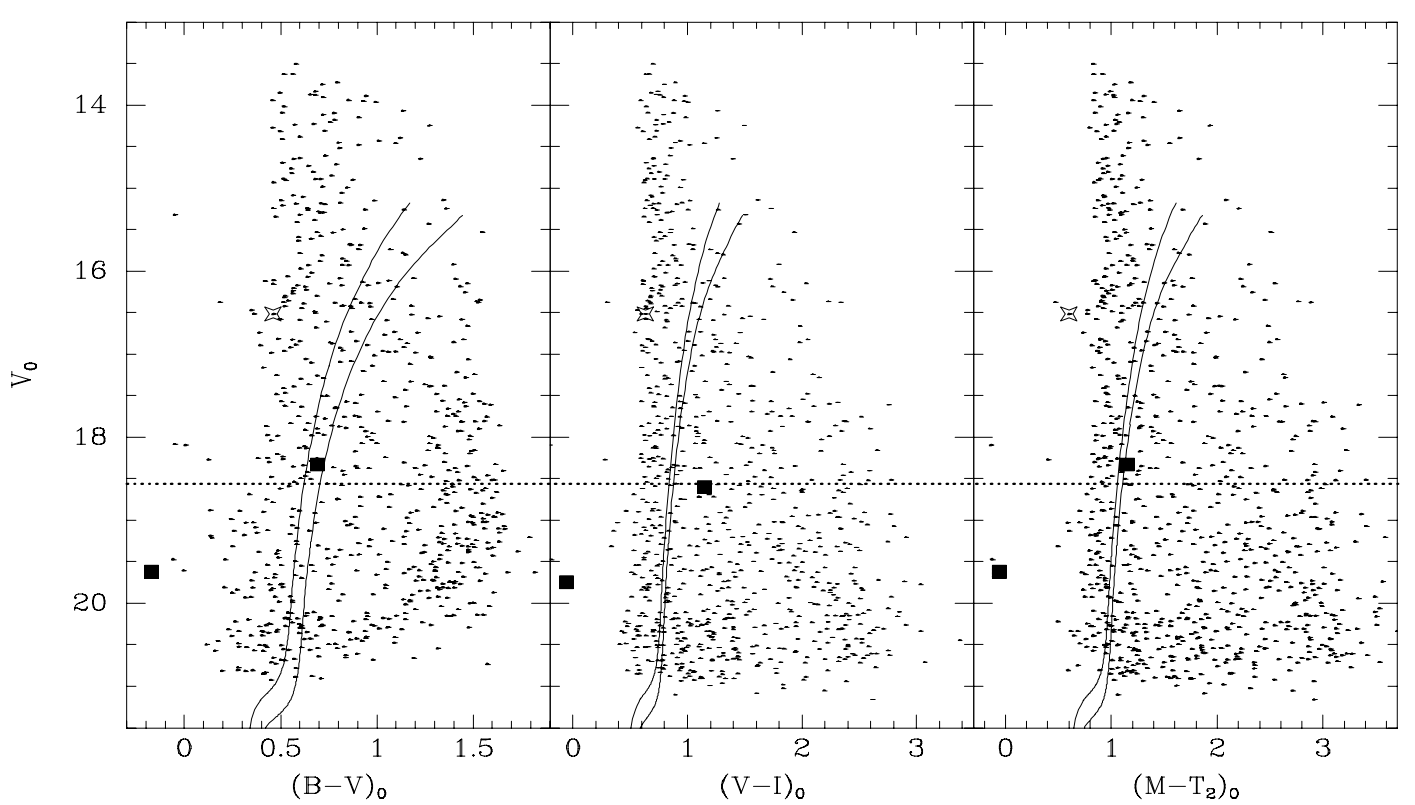

Figure 8. $B V, V I$, and $M T_{2}$ CMDs of the Willman 1 field. Stars are selected to have $-0.1<$ sharp $<0.1$ and $\sigma_{V}, \sigma_{T_{2}}, \sigma_{M}<0.15$. The dotted line indicates the hypothetical magnitude of the HB. The starred point is the variable star identified in Section 3.2; the squared points the BHB star identified in W05 and the RGB star identified in Section 3.1. The solid lines are theoretical isochrones from Dotter et al. (2007). Note the lack of any apparent RGB or BHB.

The simplest and most likely explanation is that the variable star is a foreground RR Lyrae star. It is possible, but less likely, that it is an anomalous cepheid (AC, Pritzl et al. 2004) associated with Willman 1 and embedded in an extended stellar distribution. The specific frequency of ACs has been shown to increase with declining mass and abundance (Mateo et al. 1995; Pritzl et al. 2004). However, Willman 1 is so small that even if the Pritzl et al. absolute magnitude-specific frequency relation were extrapolated over another $6 \mathrm{mag}$, it would predict less than one AC. The abundance-specific frequency relation would predict zero ACs, whether evaluated at $[\mathrm{Fe} / \mathrm{H}]=-1.5$ or -2.2 .

\subsection{The Extended Structure of Willman 1}

Figure 8 shows the $B V, V I$, and $M T_{2}$ CMDs of the Willman 1 field. These diagrams have been cleaned to retain only objects with DAOPHOT sharp parameters between -0.1 and 0.1 and photometric errors below $0.15 \mathrm{mag}$. The HB magnitude and isochrones are identical to those in Figure 6. The starred point indicates the variable star identified in Section 3.2 while the squares indicate the blue horizontal branch (BHB) star identified in W05 from SDSS and the RGB star we confirm in Section 3.1. Consistent with previous investigations and Section 3.1, we find little evidence of a prominent RGB in the $0.6 \mathrm{deg}^{2}$ PFC field. There is a slight overdensity where the lower part of the Willman 1 RGB is located. However, these faint blue objects show a flat spatial distribution across the field and are therefore more likely to represent the metal-poor turn-off of the halo-field stars or compact background galaxies.

The CMDs show about a dozen stars slightly bluer than the field star edge with $V-I, B-V$ and $M-T_{2}$ colors similar to BHB stars. A handful of these are clumped at the faint end of the data near $\mathrm{V} \sim 19.4$ and could potentially be a diffuse Willman $1 \mathrm{HB}$ if the distance modulus has been badly underestimated. However, the number and magnitude distribution of these objects would also be consistent with the number and magnitude distribution of faint blue field stars we have seen in similar surveys of other dSph galaxies such as Bootes (Siegel 2006). They also do not show any central concentration in the field.

That these faint blue objects are not Willman $1 \mathrm{HB}$ stars is demonstrated in Figure 9, which compares the raw VI field CMD to one overlayed with both isochrones and synthetic horizontal branches (SHB) from Dotter et al. (2007). The two SHBs have 50 stars each and are set at $[\mathrm{Fe} / \mathrm{H}]=-1.5$ and $-2.2,[\alpha / \mathrm{Fe}]=+0.0$. The HB mass is set at the mass of the tip of the RGB (TRGB) for the relevant isochrone with an average mass loss of $0.05 \pm 0.05 M_{\odot}$.

The apparent sequence of faint blue stars is well below the magnitude of the SHB and has a downward slope that is not seen in the synthetic model. In fact, only three objects are within 0.5 mag of the SHB. The BHB star identified in W05 lies slightly bluer and brighter than the SHB. As noted in Section 3.1, this star is at the Willman $1 \mathrm{RV}$. Its location above the SHB could indicate either that Willman 1 is closer than $40 \mathrm{kpc}$ or that the star is evolving away from the BHB. A second star is within $7^{\prime}$ the Willman 1 photocenter and along the metal-poor SHB but has a $V-I$ color of 0.28 . Our studies of other dSph galaxies (see, e.g., Siegel 2006) have shown this color to lie near or in the instability strip. Since this star does not vary, it is unlikely that it is a Willman $1 \mathrm{HB}$ star. Finally, a third star, located more than $14^{\prime}$ from the Willman 1 photocenter, is slightly brighter and bluer than the W05 HB star. While this star could be a Willman 1 member, its membership would require that the star be both extratidal and unusually blue and evolved. It is highly unlikely that an object as faint as Willman 1 has two such rare BHB stars. In short, it is likely that Willman 1 does not have any BHB stars within the PFC field beyond the one identified in W05. Willman 1 could, however, have non-variable RHB stars lost within the field star blue edge.

When combined with the negative detection of extratidal giants (Section 3.1) or RR Lyrae (Section 3.2), this indicates that our thorough $0.6 \mathrm{deg}^{2}$ survey identifies no measurable extended structure in Willman 1. Not a single confirmed RGB, RR Lyrae 


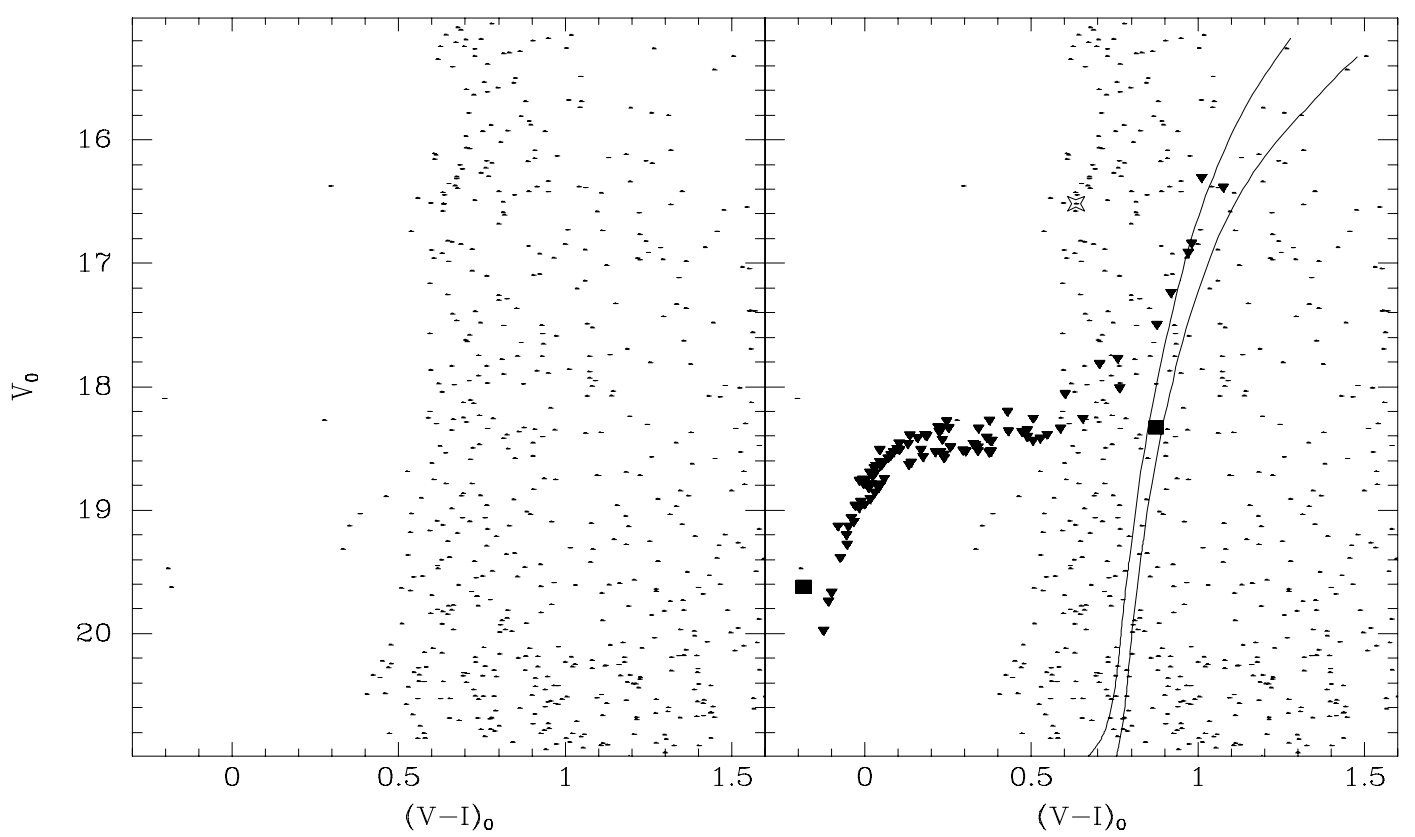

Figure 9. The raw VI CMDs of the Willman 1 field (left) and the same CMD overlayed with isochrones and synthetic horizontal branches from Dotter et al. (2007) corresponding to the possible Willman 1 populations. Symbols are as in Figure 8.

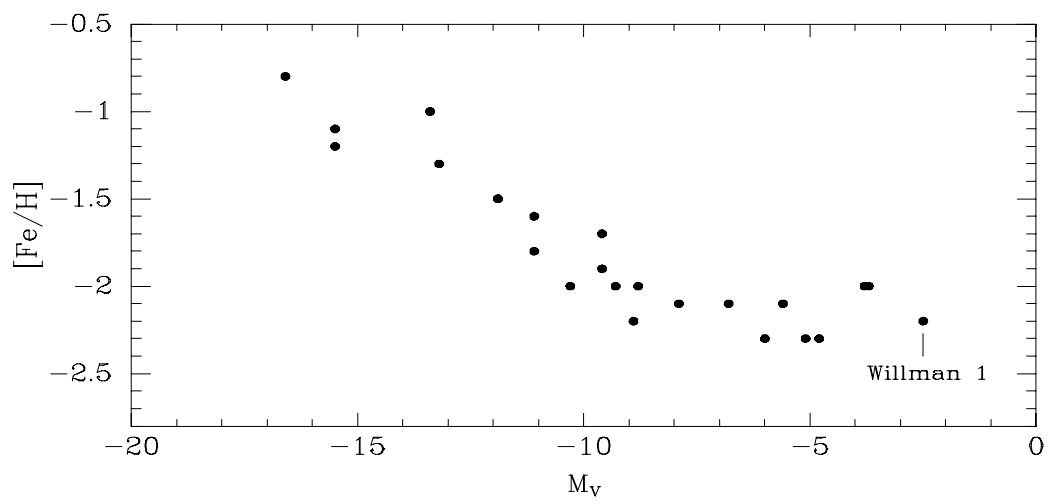

Figure 10. The revised $M_{V}-[\mathrm{Fe} / \mathrm{H}]$ relation for dSph galaxies, assuming star 1578 indicates the true abundance of Willman 1. Contrasted against Figure 1, Willman 1 's abundance is now consistent with dSph galaxies of similar luminosity.

or BHB star lies beyond the measured optical limit. The only confirmed members are the W05 HB star and some of the M05 giants, all of which are within $5^{\prime}$ of the Willman 1 photocenter.

\section{DISCUSSION AND CONCLUSIONS}

The paucity of bright spectroscopic targets in Willman 1 and the difficulty in using RVs alone to separate out members makes conclusions about its nature and properties tentative. However, our survey results have several important implications about the nature of Willman 1.

1. The metallicity spread implied by M07 is now less certain. The most metal-rich M07 star, and the only metal-rich member with $\mathrm{S} / \mathrm{N}>13$ in M07, is clearly a foreground dwarf star. Another metal-rich M07 star, 1302, is close to the dwarf locus, with an RGB probability of 34\%. Without star 1269, the metallicity dispersion of Willman 1 drops to 0.3 dex, which is only slightly greater than the measurement error (estimated by M07 to be 0.2 dex at $\mathrm{S} / \mathrm{N}=15$ ). Moreover, the remaining metal-rich Willman 1 members are close to the MSTO where the CaT abundance method used by M07 it not very reliable since it is designed for use with latetype RGB stars (Rutledge et al. 1997; Carretta \& Gratton 1997). In fact, the only confirmed member of Willman 1 with an M07 $\mathrm{S} / \mathrm{N}>15$ has an abundance of $[\mathrm{Fe} / \mathrm{H}]=$ -2.1 , which we confirm directly from the Fe lines (as $[\mathrm{Fe} / \mathrm{H}]=-2.2)$. The existing evidence does not support an abundance spread in Willman 1 and hints at a low, rather than intermediate, abundance. Figure 10 shows the revised $M_{V}-[\mathrm{Fe} / \mathrm{H}]$ trend of the dSph galaxies, assuming a -2.2 metallicity for Willman 1 and shows that a metalpoor Willman 1 would be consistent with the trend established in other very low-luminosity dSph galaxies (Muñoz et al. 2006; M07, SG07). The flattening of the luminositymetallicy relation - and many of the brighter dSph galaxies with multiple populations having similar trace metal-poor populations - could support the hypothesis of a common origin for many of the dSph galaxies (Kunkel \& Demers 1977; Lynden-Bell 1982; Majewski 1994; Lynden-Bell \& Lynden-Bell 1995; Metz \& Kroupa 2007; Metz et al. 2008). 
Alternatively, this could be a threshold metallicity for the formation of stellar populations.

2. Our study does not affect the inferred velocity dispersion of Willman 1. The nine member stars we match to our Washington photometry have a velocity dispersion of $4.4 \pm$ $1.3 \mathrm{~km} \mathrm{~s}^{-1}$. When star 1269 is removed, the remaining M07 stars have a dispersion of $4.5 \pm 1.5 \mathrm{~km} \mathrm{~s}^{-1}$. Further pruning of the sample-even down to the extreme of the three stars that are either spectroscopically or photometrically classified as giants - does not reduce the velocity dispersion.

3 . We measure a slightly sub-solar $\alpha$-abundance in star 1578 , a value similar to but lower than the $\alpha$-abundances measured in stars of similar $[\mathrm{Fe} / \mathrm{H}]$ in other dSph galaxies (Venn et al. 2004) and globular clusters thought to have been tidally stripped from dwarf galaxies (e.g., Palomar 12; Dinescu et al. 2000; Cohen 2004). This indicates that Willman 1 has a chemical evolution history similar, but not identical, to that of other dwarf galaxies. It certainly formed in a different environment than the Milky Way field stars or globular clusters. A slow chemical evolution would be consistent with Willman 1 having always been a low-mass object.

4. While the isophotes of W07 appear to show an irregular object, the wide field $0.6 \mathrm{deg}^{2}$ survey of Willman 1 fails to conclusively identify a single bright RGB star, a single RR Lyrae star or a single BHB star outside the half-light radius (1'.9). In fact, we identify no Willman 1 members beyond those identified in M07 (several of which appear to be non-members). The lack of extratidal stars supports the structural analysis of M07 and W07, which, using the more numerous stars of the upper MS, show radial profiles that are roughly consistent with a King (1962) or exponential profile with no indication of extended structure.

5. It is important, however, to realize that the extant data are limited in their ability to probe the extended structure of Willman 1. W07 and M07 only trace Willman 1 over two orders of magnitude and barely reach the foreground star count level in the outer isophotes. Such surveys can lack the sensitivity needed to trace low-surface-brightness extended structure (see, e.g., Siegel et al. 2008). While tracer studies of evolved stars are more sensitive to lowsurface-brightness extensions, Willman 1 is so small and sparse that even the DDO51 photometry provides no additional information on the surface brightness profile. Only a deeper survey using an $8 \mathrm{~m}$ class imager would be able to trace Willman 1's outer isophotes to fainter levels using faint MS stars.

These properties are consistent with a low-mass dark-matterdominated dSph galaxy. However, it is worth noting that they would also be consistent with a low-mass globular cluster in the late stages of tidal disruption by the Milky Way. Willman 1 bears some resemblance to the globular cluster Palomar 13. Pal 13, like Willman 1, is small, diffuse and shows signs of being tidally disrupted (Siegel et al. 2001). Côté et al. (2002) measured an apparent $\mathrm{M} / \mathrm{L}$ of 40 for Pal 13. While this can be interpreted as an indication of dark matter, objects in the final throes of tidal disruption can also have an inflated core velocity dispersion (Muñoz et al. 2007). Moreover, Blecha et al. (2004) and Clark et al. (2004) have shown that Palomar 13 has a high binary fraction, possibly resulting from the preferential loss of lowmass stars, which inflates the measured velocity dispersion. This is an important point to keep in mind with Willman 1, which, like Palomar 13, could have an inflated velocity dispersion owing to its overlap with the disk RV distribution, its sparseness and/or a high binary fraction. A binary fraction enhanced by mass loss would also account for the broad MS observed in M07 and W07, obviating the need for an abundance spread.

Determining whether Willman 1 is a low-luminosity dSph or a disrupting globular cluster remains just out of reach of the present studies but is amenable to further investigation. Deep spectroscopy from next-generation telescopes would allow abundances to be measured for the MSTO and MS stars, minimizing the problem of foreground dwarf contamination. Further spectroscopy and more extensive photometry would reveal if Willman 1, like Palomar 13, has a high binary fraction that is inflating its velocity dispersion. Proper motions would allow separation of member stars from the field and determine the orbit of Willman 1, revealing much about its dynamical past.

As more low-luminosity objects are discovered in the SDSS, the parameter space defined by these objects will become clearer. For the moment, Willman 1 still remains in the nebulous parameter space between dSph galaxies and globular clusters. It has yet to definitively reveal its nature.

Support for this work was provided by NSF grant AST0306884. We would like to thank Steve Odewahn, Eusebio Terrazas, John Caldwell, Heinz Edelmann, Vicki Riley, and Frank Deglman for their assistance in collecting the HET spectra. The HET is a joint project of the University of Texas at Austin, the Pennsylvania State University, Stanford University, Ludwig-Maximilians-Universität München, and Georg-AugustUniversität Göttingen. The HET is named in honor of its principal benefactors, William P. Hobby and Robert E. Eberly.

\section{REFERENCES}

Adelman-McCarthy, J. K., et al. 2006, ApJS, 162, 38

Bessell, M. S. 2001, PASP, 113, 66

Blecha, A., Meylan, G., North, P., \& Royer, F. 2004, A\&A, 419, 533

Carretta, E., \& Gratton, R. G. 1997, A\&AS, 121, 95

Clark, L. L., Sandquist, E. L., \& Bolte, M. 2004, AJ, 128, 3019

Cohen, J. G. 2004, AJ, 127, 1545

Côté, P., Djorgovski, S. G., Meylan, G., Castro, S., \& McCarthy, J. K. 2002, ApJ, 574,783

Dinescu, D. I., Majewski, S. R., Girard, T. M., \& Cudworth, K. M. 2000, AJ, 120,1892

Dotter, A., Chaboyer, B., Jevremovic, D., Baron, E., Ferguson, J. W., Sarajedini, A., \& Anderson, J. 2007, AJ, 134, 376

Fellhauer, M., et al. 2007, MNRAS, 375, 1171

Geisler, D. 1990, PASP, 102, 344

Geisler, D. 1996, PASP, 111, 480

Hinkle, K., Wallace, L., Valenti, J., \& Harmer, D. 2000, in Visible and Near Infrared Atlas of the Arcturus Spectrum 3727-9300 ̊, ed. K. Hinkle, L. Wallace, J. Valenti, \& D. Harmer (San Francisco, CA: ASP)

King, I. R. 1962, AJ, 67, 471

Kuhn, J. R., Smith, H. A., \& Hawley, S. L. 1996, ApJ, 469, L93

Kunkel, W. E., \& Demers, S. 1977, ApJ, 214, 21 (KD77)

Landolt, A. U. 1992, AJ, 104, 340

Layden, A. C. 1998, AJ, 115, 193

Lynden-Bell, D. 1982, Observatory, 102, 202

Lynden-Bell, D., \& Lynden-Bell, R. M. 1995, MNRAS, 275, 429

Majewski, S. R., Ostheimer, J. C., Kunkel, W. E., \& Patterson, R. J. 2000a, AJ, 120,2550

Majewski, S. R., Ostheimer, J. C., Patterson, R. J., Kunkel, W. E., Johnston, K. V., \& Geisler, D. 2000b, AJ, 119, 760

Majewski, S. R., et al. 1994, ApJ, 431, 17L

Martin, N. F., Ibata, R. A., Chapman, S. C., Irwin, M., \& Lewis, F. G. 2007, MNRAS, 380, 281 [M07]

Mateo, M. 1998, ARA\&A, 36, 435

Mateo, M., Fischer, P., \& Krzeminski, W. 1995, AJ, 110, 2166

Metz, M., \& Kroupa, P. 2007, MNRAS, 376, 387

Metz, M., Kroupa, P., \& Libeskind, N. I. 2008, ApJ, arXiv:0802.3899 
Muñoz, R. R., Carlin, J. L., Frinchaboy, P. M., Nidever, D. L., Majewski, S. R., \& Patterson, R. J. 2006, ApJ, 650, L51

Muñoz, R. R., Majewski, S. R., \& Johnston, K. V. 2007, ApJ, submitted

Palma, C., Majewski, S. R., Siegel, M. H., Patterson, R. J., Ostheimer, J. C., \& Link, R. 2003, AJ, 125, 1352

Paltoglou, G., \& Bell, R. A. 1994, MNRAS, 268, 793

Pritzl, B. J., Armandroff, T. E., Jacoby, G. H., \& Da Costa, G. S. 2004, AJ, 127,318

Ramirez, I., \& Melendez, J. 2005, ApJ, 626, 465

Robin, A. C., Reyle, C., Derriere, S., \& Picaud, S. 2003, A\&A, 409,523

Rutledge, G. A., Hesser, J. E. \& Stetson, P. B. 1997, PASP, 109, 907

Schlegel, D. J., Finkbeiner, D. P., \& Davis, M. 1998, ApJ, 500, 525

Shetrone, M., Venn, K. A., Tolstoy, E., Primas, F., Hill, V., \& Kaufer, A. 2003, AJ, 125, 684

Shetrone, M., et al. 2007, PASP, 119, 556

Siegel, M. H. 2006, ApJ, 649, L83

Siegel, M. H., Majewski, S. R., Cudworth, K. M., \& Takamiya, M. 2001, AJ, 121,935
Siegel, M. H., Majewski, S. R., Reid, I. N., \& Thompson, I. B. 2002, ApI, 578,151

Siegel, M. H., Majewski, S. R., Sohn, S. T., Patterson, R. J., Shetrone, M. D., \& Muñoz, R. R. 2008, ApJ, submitted

Simon, J. D., \& Geha, M. 2007, ApJ, 670, 313

Skrutskie, M. F., et al. 2006, AJ, 131, 1163

Sohn, S. T., et al. 2007, ApJ, 663, 960

Stetson, P. B. 1987, PASP, 99, 191

Stetson, P. B. 1990, PASP, 102, 932

Tull, R. G. 1998, Proc. SPIE, 3355, 387

Venn, K. A., Irwin, M., Shetrone, M. D., Tout, C. A., Hill, V., \& Tolstoy, E. 2004, AJ, 128, 1177

Westfall, K. B., Majewski, S. R., Ostheimer, J. C., Frinchaboy, P. M., Kunkel, W. E., Patterson, R. J., \& Link, R. 2005, AJ, 131, 375

Willman, B., et al. 2005, AJ, 129, 2692, [W05]

Willman, B., et al. 2007, AJ, submitted (arXiv astro-ph/0603486) [W07]

Zacharias, N., Monet, D. G., Levine, S. E., Urban, S. E., Gaume, R., \& Wycoff, G. L. 2004, BAAS, 205.4815

Zucker, D. B., et al. 2006, ApJ, 659, L41 